\title{
CONTROLLING FIELD QUALITY IN MAGNET PRODUCTION
}

\author{
L. Bottura, A. Devred, V. Remondino, S. Sanfilippo, W. Scandale, E. Todesco, C. Vollinger, \\ E. Wildner, CERN, Geneva 1211, Switzerland
}

\begin{abstract}
The field quality measurements at room temperature of around 100 collared coils of the main LHC dipoles are analysed. Using correlations to field measurements at $1.9 \mathrm{~K}$, comparison with beam dynamics limits are discussed. Both random and systematic components are analysed, allowing to pin out the most critical multipoles. Corrective actions that have been taken during the preseries phase are presented; we focus on the low order systematic multipoles that are shown to be the most difficult components to steer. A preliminary analysis of the integrated main field and of its implications on the possible scenarios for the installation are also carried out.
\end{abstract}

\section{INTRODUCTION}

Magnetic measurements are carried out with a $75 \mathrm{~mm}$ long rotating coil on 20 consecutive positions in longitudinal direction to cover the $15 \mathrm{~m}$-long LHC dipoles. For each position, the field harmonics up to order 15 are measured, in both apertures. Each harmonics averaged over the dipole axis is compared to beam dynamics requirements, whilst spikes in the longitudinal profile help in detecting local defects during assembly.

In this paper we will discuss our approach for quality control, identify targets for field shape, present limits to corrective actions during production and finally examine the status of field quality in the pre-series LHC dipoles. We will express units in $10^{-4}$, at $17 \mathrm{~mm}$ reference radius.

\section{QUALITY CONTROL}

In order to fix field-shape ranges for quality control, for each manufacturer, we select a sufficiently large group of data and we separate them into three sets: straight part, connection and non-connection heads. We compute the average and the $\sigma$ for each set, rejecting cases clearly out of statistics [1], and we set control limits at $\pm 3.5 \sigma$ (warning) and at $\pm 7 \sigma$ (alarm). For Gaussian distributions, we should expect a single warning during the production. We use the same approach both for collared coils and for the cold mass. In the latter case, for a more stringent test, we compute the difference from collared coil harmonics, since the contribution of the iron yoke is very stable [2].

Automatic checks of magnetic measurements allowed us to detect both faulty measurements (4 over 98 ) and assembly errors ( 2 over 98$)$. We found the first error in collared coil 2002, having a big spike of 40 units in the main field, of 24 units in $b_{2}$, plus minor spikes in $a_{2}, a_{3}$ and $b_{3}$ along a length of $2 \mathrm{~m}$ in the central part. This was induced by the erroneous insertion of a second coil protection sheet, $0.5 \mathrm{~mm}$ thick, in the corresponding dipole section. We found the second error in collared coil 1027 , showing a localized spike in $b_{2}$ of 5.5 units, with minor anomalies in $b_{3}$ and $a_{2}$. This was induced by a missing shim, $0.8 \mathrm{~mm}$ thick, along a length of $1.5 \mathrm{~m}$, in the pole outer layer. We could predict its location by field simulations considering the sign of the faulty harmonics.

\section{FIELD QUALITY TARGETS}

Targets for field shape harmonics are given in terms of systematic (average along the ring), random ( $\sigma$ for one arc - a priori the same in each arc) and uncertainty ( $\sigma$ of the average per arc) [3]. Random and uncertainty targets result from a compromise between what is needed for beam stability and what can be reached with realistic mechanical tolerances. The final choice mostly relies on experience of previous machines [4,5]. Systematic targets and their acceptance ranges, instead, result from beam dynamics considerations only: any target is achievable by the design of the coil cross-section, whilst keeping it within the allowed range for the whole production is the true challenge. Ranges for systematic harmonics at injection and at high energy differ by an offset resulting from persistent currents, iron saturation and electromagnetic forces [6]. Their intersection determines the effective range for field quality. Finally, targets and ranges for collared coil harmonics result from correlation of harmonics measured in warm and in cold conditions.

The criticality of each harmonic is proportional to the ratio between its measured spread during production and the allowed range for the systematic value. In Table 1, lower order harmonics are evaluated for already produced collared coils. With our criterion, $b_{5}, a_{2}$ and $a_{4}$ are the most critical, whilst $b_{3}, b_{4}$ and $a_{3}$ are the easiest to control.

Table I: Measured random component versus the allowed range for the systematic, given in Ref. [3].

\begin{tabular}{lllllllll}
\hline & $\mathrm{b}_{3}$ & $\mathrm{~b}_{5}$ & $\mathrm{~b}_{7}$ & $\mathrm{~b}_{2}$ & $\mathrm{~b}_{4}$ & $\mathrm{a}_{2}$ & $\mathrm{a}_{3}$ & $\mathrm{a}_{4}$ \\
\hline$\sigma$ & 1.50 & 0.45 & 0.17 & 0.70 & 0.11 & 1.30 & 0.40 & 0.30 \\
range & 7.00 & 0.70 & 0.48 & 2.20 & 0.74 & 2.00 & 1.40 & 0.28 \\
\hline
\end{tabular}

\section{LIMITS TO CORRECTIVE ACTIONS}

The time sequence of the dipole production shows that we should take corrective actions already on collared coils in order to shorten the feedback delay. A correlation between measurements at $300 \mathrm{~K}$ in the industry and in cold operational conditions is needed for an effective steering of the field quality.

A predictive magnetic model is another key tool, since forecasting absolute values of field harmonics is essential in conceiving prototypes. With our numerical model which also evaluates iron contributions and coil deformations [6-9], we obtained an agreement between measured and expected values of 1 to 2 times the measured random component. Indeed, $b_{3}$ agrees within 3 
units, $b_{5}$ within 1 unit and $b_{7}$ within 0.4 units. If deformations are neglected, the agreement on $b_{5}$ becomes much worse ( $4 \sigma$, i.e. 2 units) [8-9]. This is however insufficient for steering the field quality, and iterations on the coil design are needed. In this case, our model is used in differential, to enhance the precision. For the LHC dipoles we carried out a comparison between model and measurements in three different cases:

- In reshaping the iron insert to reduce the systematic values of $b_{2}$ and $b_{4}$ [10].

- In resizing the polar shims to optimize the systematic values of $b_{3}, b_{5}$ and $b_{7}[11,12]$.

- In choosing the size of the mid-plane insulation to optimize the systematic values of $b_{3} b_{5}$ and $b_{7}$ [13].

The agreement, always found within $20 \%$, is considered excellent. Indeed, just to evaluate the effect of a coil deformation we may use a different realistic hypothesis, which may already lead to differences up to $10 \%$.

In comparing the harmonics of the collared coils with cold measurements we observe a linear correlation with a slope close to the theoretical value of $1 / 1.18$, induced by the iron yoke effect of $18 \%$ on the main field [14]. The spread around the linear fit is to be compared to the allowed ranges for systematic components [3]. This spread is large only for $a_{2}, a_{4}$ and $b_{5}$. On the other hand, the influence of persistent currents on $b_{3}, b_{5}$ and $b_{7}$ seems to be well understood and reproducible.

\section{FIELD QUALITY STATUS}

\section{Systematic harmonics}

All systematic skew and even normal harmonics are within targets. The corrective action taken at the end of the prototype phase on the insert shape to correct $b_{2}$ and $b_{4}$ [10] has been effective. The odd normal harmonics are shown in the right part of Fig. 1, and are still too large for the second cross-section.
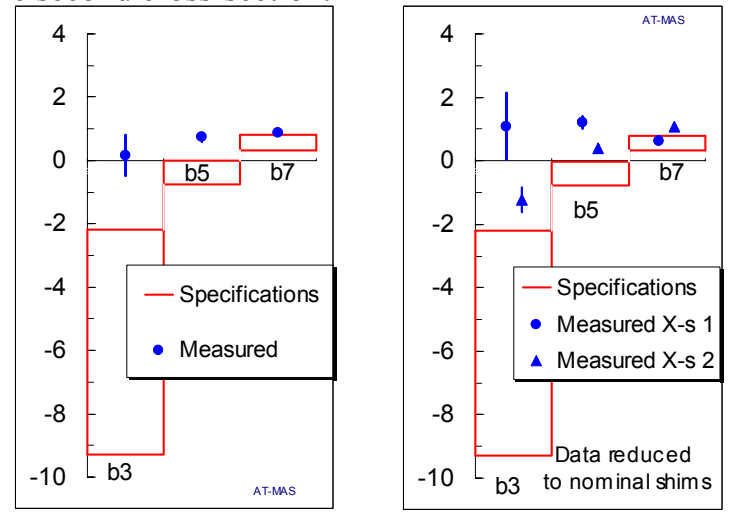

Figure 1: Systematic $b_{3}, b_{5}$ and $b_{7}$ (blue dots) versus beam dynamics targets (red), raw data (left) and data separated by cross-section, reduced to nominal shims (right).

In order to reduce $b_{3}$ and $b_{5}$, we decided on a reshaping of the internal copper wedges by less than $0.4 \mathrm{~mm}$, keeping the same coil and collar shape [15]. Our aim was shifting $b_{3}$ by -3.5 units, $b_{5}$ by -1.35 units and $b_{7}$ by
+0.18 units. The observed effect instead was -3 units for $b_{3},-0.80$ units for $b_{5}$ and +0.45 for $b_{7}$. We decided to change the cross section after 9 collared coils. Afterwards we saw an unexpected trend of 7 units of $b_{3}$ in the first 15 collared coils. We could explain only a part of the trend (2 units) by out-of-tolerance copper wedges [16].

\section{Random harmonics}

In Fig. 2, we show random harmonics in collared coils, reduced to nominal shims. Data relative to the first crosssection shows larger spreads than in the second crosssection, due to a learning process during the production. The random imperfections are well within specifications or at the limit as for the integrated main field $\mathrm{BdL}$, for $b_{2}$ and for $b_{5}$. Only $b_{3}$ is out-of-tolerance because of the trend in first cross-section. This makes the installation scenario of a full dipole mixing compatible with beam dynamics targets from the point of view of geometric harmonics.

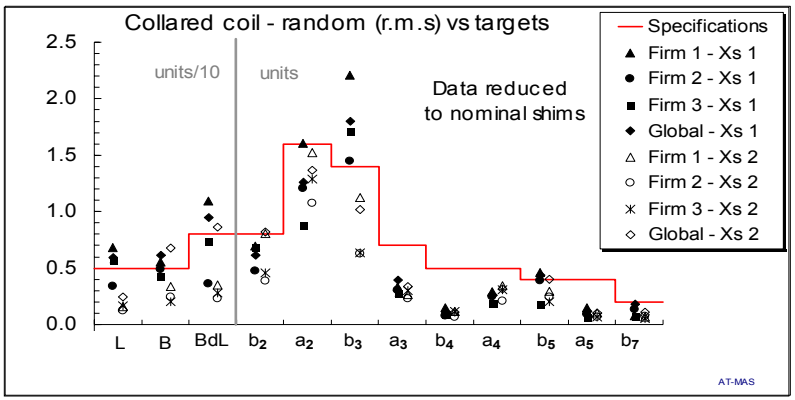

Figure 2: Random multipoles: targets versus measured values per manufacturer and for the full production, separated by cross-sections, reduced to nominal shims.

\section{First estimates of uncertainty}

We observe a non-negligible difference between manufacturers in the following quantities:

- Main field: approx. 20 units difference between Firm3 and the other Firms.

- $b_{2}$ : 1.5 units smaller (larger) value in aperture 1 (aperture 2) for Firm3 than elsewhere, probably due to different collar manufacturers.

- $b_{5}: 1$ unit of differences between Firm1 and Firm2, Firm3 being in between.

- $b_{7}$ : up to 0.4 units of difference between Firm1 and Firm2, Firm3 being in between.

Magnetic length, $b_{3}, b_{4}$ and skews show no systematic differences between manufacturers.

\section{OPEN POINTS}

\section{Systematic odd normal multipoles}

The present large values of systematic $b_{5}$ and $b_{7}$ seem to have a negligible impact on beam stability. However, in case of a drift, these multipoles can go out-of-tolerance. Moreover, at high field, $b_{3}$ should be corrected using chromatic sextupoles, thereby reducing operational flexibility. In order to reduce $b_{3} b_{5}$ and $b_{7}$ a solution is to 
increase the thickness of the insulation in the coil midplane [17]. An additional layer of $0.1 \mathrm{~mm}$ insulation would recover safer values for these multipoles, with a minimal cost. A test on a $1 \mathrm{~m}$-long model is in progress, showing results in agreement with models [13].

\section{Systematic differences in integrated main field}

In collared coils 30 to 80 , the integrated main field is systematically larger by about 20 units in Firm3, as shown in Fig. 3. This brings the random $B d L$ at the limit of the target and stem in a variation of the main field in the straight part, whilst the magnetic length does not show relevant differences between manufacturers. A difference of $56 \mu \mathrm{m}(0.2 \%)$ in the coil radius can explain it. Instead, nor copper wedge dimension errors, neither differences in collar manufacturers (contrary to the case of $b_{2}$ ) can explain this difference. We can correct the effect by acting on ferromagnetic laminations to compensate with a higher or lower magnetic length the offset in the main field. A decrease of magnetic length in Firm 3 and an increase elsewhere according to the maximal values given in the dipole specification should allow to compensating half of this systematic offset. Data in operational conditions partially confirm this offset, but more statistics would be desirable before carrying out the corrective action.

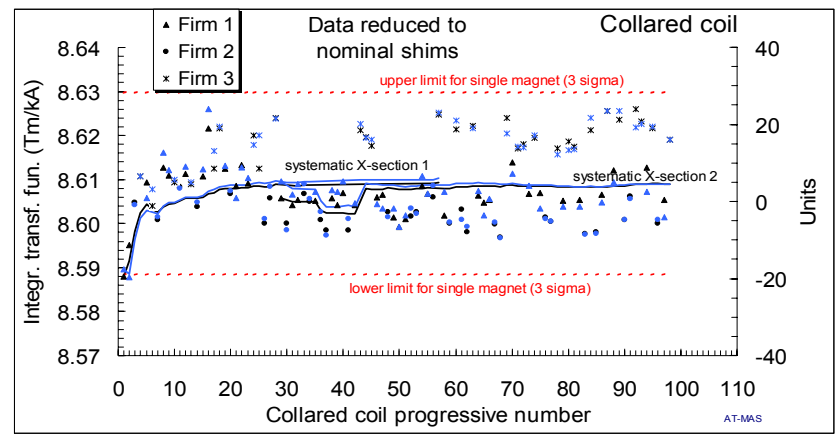

Figure 3: Integrated main field in the collared coil: measured data (markers) and $\pm 3 \sigma$ limit (dotted lines). Data reduced to nominal shims.

\section{Field quality variation after re-collaring}

Four magnets have been re-collared for electrical problems or assembly faults. Field quality measurements showed a non-negligible systematic variation of odd normal multipoles: the larger effect is a positive change of $b_{5}$ of around 0.5 units. This could be due to a plastic deformation of the coil or maybe of the collars. Indeed, this should not play a relevant role in the field quality control since we expect to collar more than once only a small fraction of collared coils.

\section{CONCLUSIONS}

The magnetic field of 98 collared coils, 56 cold masses and 24 cryomagnets has been measured. Magnetic measurements at room temperature are being successfully used as a tool to detect faulty components or assembly procedures. Two collared coils have been intercepted for anomalies in magnetic field, and in both cases assembly errors have been found. The automatic procedures to screen magnetic measurements are active. Measured random harmonics are within specifications for beam dynamics [3]. The hard part is steering systematic harmonics, like $a_{2} a_{4}$ and $b_{5}$ where the allowed range is small compared to the random part.

Our capability of modelling field quality in the collared coil is rather good [10]. Sensitivities matrices can be estimated with $20 \%$ error. The absolute agreement between model and measurements is within two times the random component, coil deformations playing a strong role for the $b_{5}$ [8-9]. The steering of field quality should be based on collared coil data, due to the delay of months between coil assembly and final tests at $1.9 \mathrm{~K}$. Correlations between collared coil data and measurements at $1.9 \mathrm{~K}$ are rather good since the contribution of persistent currents, saturation and Lorentz forces is well reproducible, but they should be carefully monitored during the whole production.

Systematic skew and even normal multipoles are within targets. For odd normal multipoles we applied corrective action on the coil cross-section [15], and the present values provide the required beam stability. However, we are at the limit of the targets for $b_{3}, b_{5}$ and $b_{7}$ hence an additional corrective action will be probably necessary.

A corrective action based on a change of the magnetic length is in preparation to compensate part of the 20 units difference in BdL between Firm3 and the other manufacturers.

\section{ACKNOWLEDGEMENTS}

We wish to thank L. Rossi and J. Billan for support, discussions and help given in this work. We acknowledge I. Vanenkov for providing data and analysis of coil size and impact on field quality. We thank D. Tommasini and his team and the project engineers of the AT-MAS group for the constant support in the analysis and for providing assembly data. Thanks to S. Fartoukh and O. Bruning for discussing the beam dynamics targets and for comments.

\section{REFERENCES}

[1] S. Pauletta, Master Thesis, Torino University (2002).

[2] E. Wildner, E. Todesco, priv. communication (2003).

[3] S. Fartoukh, O. Bruning, LHC Pr. Rep. 501 (2001).

[4] L. Walckiers, private communication (1999).

[5] L. Walckiers et al, EPAC (2002) 260-2.

[6] R. Gupta et al., EPAC (1994) 2298-300.

[7] S. Russenschuck, LHC Project Report 276 (1999).

[8] P. Ferracin et al, IEEE Tr. Appl. Supercon. 12 (2001)

[9] P. Ferracin, Ph.D. Thesis, Torino University (2001).

[10] P. Ferracin et al., LHC Project Report 467 (2001).

[11]Z. Ang et al, IEEE Tr. Appl. Supercon. 10 (1999).

[12] P. Ferracin et al, Phys. Rev. STAB, 5 (2002).

[13] cern.ch/lhc-div-mms/MMSPAGES/MA/mid_ins.html

[14]P. Tollestrup, Fermilab Report UPC-86 (1979).

[15] E. Todesco et al, EPAC (2002) 2439-41.

[16] B. Bellesia et al, LHC Pr. Rep. (2003) in press.

[17] L. Bottura, internal memorandum, March 2001. 